\title{
Saturation of red: A prothetic continuum'
}

D. W. PANEK AND S. S. STEVENS

HARVARD UNIVERSITY

A battery of procedures was used to scale the saturation of colors produced by mixtures of red and gray papers. By direct magnitude estimation, the apparent saturation was found to grow as the 1.7 power of the percentage of red in the mixture. The power law was confirmed by the cross-modality matching of loudness to saturation. The inverse continuum, paleness, was also scaled.

Saturation appears to be a prothetic continuum because the category scale is curved and the subjective size of the jind increases as saturation increases.

The purpose of these experiments was to determine the quantitative relation between the purity of a hue and the subjective impression of its saturation or desaturation. Among other things, it was hoped that the application and comparison of several kinds of scaling procedures would answer the question whether saturation is a metathetic of a prothetic continuum, i.e., whether it behaves as a qualitative or a quantitative variable.

On the basis of the evidence then available, an answer to that question was conjectured in 1939 (Stevens, 1939; Stevens \& Volkmann, 1940). It now appears that the guess was wrong, and that saturation is not a metathetic continuum. On present evidence, saturation must be numbered among the prothetic continua, a class that includes such intensive attributes as brightness and loudness. Saturation lacks the wide dynamic range of brightness and loudness, however, and consequently it is less easy to apply the tests needed to produce a firm decision about the nature of the continuum. If saturation is prothetic, its apparent magnitude should grow as a power function of per cent purity, and the subjective size of the just noticeable difference (jnd) should grow approximately in proportion to the subjective magnitude. These are two of the defining criteria of a prothetic continuum (Stevens, 1957). It should be noted, however, that the power-function form is not a sufficient condition to determine that a continuum is prothetic.

The experiments were begun in 1961 after it became clear that the pioneering study by Galifret (1959) had left the issue in some respects unresolved. A series of studies by Onley et al (1963) has shown that for both red and green light the apparent saturations grow as power functions of purity with exponents in the vicinity of 2.0 .

\section{Apparafus}

A cylindrical color mixer, similar in principle to that used by Galifret (1959), provided target stimuli of various saturations. This color mixer is a rotating cylinder, $45 \mathrm{~cm}$ long and $4 \mathrm{~cm}$ in diameter, on which are placed colored and gray papers in such a way that the mixture is completely gray at one end of the cylinder and completely colored at the other, with a linear change in purity from one end of the cylinder to the other. The color mixer was rotated at about $2000 \mathrm{rpm}$ and was illuminated from above by a 60watt incandescent bulb in front of which was mounted a milk-glass diffuser $15 \mathrm{~cm}$ square. The milk glass was positioned $23 \mathrm{~cm}$ above the axis of the cylinder. The observer viewed the cylinder of the color mixer monocularly through a $30-\mathrm{cm}$ reduction tube which provided a 2.8-degree field. Since the axis of the viewing tube was perpendicular to the axis of the color mixer, a stimulus of the desired purity could be presented merely by moving the cylinder to the appropriate position. The time of exposure was controlled by a shutter at the end of the viewing tube. The luminance of the stimulus, as seen by the observer, was about $78 \mathrm{db}$ re $10^{-10}$ lambert, or approximately $6 \mathrm{~mL}$. The reference level $10^{-10} \mathrm{~L}$ is close to the absolute threshold of the eye.

The colored papers used were Color-Aid Red Hue, mixed with Color-Aid No. 5 Gray (from Color-Aid Co., New York, N.Y.). This gray has a reflectance of about 20 per cent. The luminance of the mixture, as observed with the aid of a Macbeth Illuminometer, did not vary noticeably with change of purity. Although the stimuli were produced by surface colors, when they were seen through the reduction tube, the impression was that of a small luminous field subtending 2.8 degrees. Although the purity of the ColorAid red is left unspecified, the resulting uncertainty does not affect the relative values produced on the color mixer. In what follows, only the relative stimulus values are used. The exponent of the power function depends only on the relative values.

The scale of apparent saturation (redness) was determined both by magnitude estimation and by crossmodality matching. Both redness and paleness were scaled. The scale of redness was compared to the results of category rating and to jnd measurements by the methods of constant stimuli and single stimuli. (These methods are discussed in Stevens, 1958, 1959, and Stevens \& Galanter, 1957.) A total of 33 observers participated. The group (usually 10 observers) for a given experiment was drawn more or less randomly from among the students and staff of the laboratory. They were tested individually, and the order in which the stimuli were presented was different for each observer. One student had a color deficiency and was not used. 
Table 1. Stimulus, geometric means, and quartile deviations for the four experiments by the method of magnitude estimation. The symbol for each experiment refers to the symbol used in Fig. 1. The quartile deviations (half the interquartile range) are expressed in decilogs, a tenth of a logarithmic unit.

\begin{tabular}{|c|c|c|c|}
\hline $\begin{array}{l}\text { Fig. } 1 . \\
\text { symbol }\end{array}$ & $\begin{array}{l}\text { Per cent } \\
\text { red }\end{array}$ & $\begin{array}{c}\text { Geometric } \\
\text { meon }\end{array}$ & $\begin{array}{c}\text { Quartile } \\
\text { deviation in } \\
\text { decilogs }\end{array}$ \\
\hline \multirow{8}{*}{ circle } & 20 & 1.5 & 2.2 \\
\hline & 30 & 3.4 & 1.7 \\
\hline & 40 & 4.7 & 1.0 \\
\hline & 50 & 7.9 & 1.0 \\
\hline & 60 & 10.8 & 0.8 \\
\hline & 70 & 12.4 & 0.7 \\
\hline & 80 & 16.1 & 1.2 \\
\hline & 90 & 19.8 & 0.8 \\
\hline \multirow{8}{*}{ triangle } & 20 & 2.1 & 1.7 \\
\hline & 30 & 3.2 & 1.1 \\
\hline & 40 & 5.7 & 1.2 \\
\hline & 50 & 7.9 & 0.9 \\
\hline & 60 & 11.6 & 0.8 \\
\hline & 65 & 13.0 & 1.0 \\
\hline & 80 & 18.7 & 0.8 \\
\hline & 100 & 30.2 & 0.8 \\
\hline \multirow{8}{*}{ square } & 10 & 69.3 & 1.6 \\
\hline & 20 & 50.8 & 1.4 \\
\hline & 30 & 32.3 & 0.7 \\
\hline & 40 & 25.2 & 0.9 \\
\hline & 50 & 18.3 & 0.9 \\
\hline & 65 & 14.2 & 0.7 \\
\hline & 80 & 8.1 & 1.6 \\
\hline & 99 & 4.3 & 2.4 \\
\hline \multirow{8}{*}{ star } & 1 & 139.2 & 1.9 \\
\hline & 20 & 68.6 & 2.1 \\
\hline & 35 & 32.4 & 1.2 \\
\hline & 50 & 19.7 & 1.0 \\
\hline & 60 & 11.8 & 1.2 \\
\hline & 70 & 8.3 & 1.1 \\
\hline & 80 & 4.8 & 1.8 \\
\hline & 90 & 3.1 & 1.7 \\
\hline
\end{tabular}

\section{Magnitude Estimation}

The procedure of magnitude estimation can be illustrated with the aid of a typical sample of the written instructions:

I am going to present a series of stimuli that vary in saturation (redness). Your task is to tell how red each stimulus appears by assigning a number to it. Call the first stimulus any number that seems to you appropriate.

To the succeeding stimuli assign numbers proportional to your subjective impression. If a stimulus appears 3 times as red, assign a number 3 times as large as the first. If it seems $1 / 5$ as red, assign a number $1 / 5$ as large, and so on.

Do you have any questions?

In some of the first experiments the observer was shown a standard, 50 per cent red, which was called 10 , but experience with the method of magnitude estimation in many contexts has shown that certain second- order biases can often be avoided by allowing the observer to choose his own modulus. The fewer the constraints placed on the observer's judgment, the better the results, provided he has grasped the basic idea of making his numerical estimates proportional to the perceived magnitude. The use of a different modulus by different observers affects only the intercept of the power function when averaging is done logarithmically, i.e., by computing geometric means.

The results of four magnitude-estimation experiments are shown in Table 1 and Fig. 1. Each point in Fig. 1 is the geometric mean of two estimates by each of ten observers. The circles represent an experiment in which a standard (50 per cent red, called 10) was presented at the beginning of the series. In the other experiment (triangles) there was no standard, and the observer called the first stimulus whatever value seemed to him appropriate. Every stimulus except the two at the extremes (20 and 100 per cent red) was presented as the first stimulus to one or another observer. In the $\log -\log$ coordinates of Fig. 1 the ordinate values of one of the experiments were adjusted to make the two sets of data points (circles and triangles) coincide approximately. Both sets of points approximate a straight line with a slope of 1.7 . Under these experimental conditions, apparent saturation grows approximately as a power function with an exponent equal to 1.7 .

The inverse of saturation is desaturation or paleness. It is an interesting question whether an observer can make consistent magnitude estimations of the inverse aspect. On nine other continua, the estimates made of the inverse aspect, e.g., dimness instead of brightness (Stevens \& Guirao, 1963), have produced fair approximations to reciprocal functions-functions with negative exponents having the same absolute values as the exponents obtained for the primary functions.

How well, or poorly, inverse scaling succeeds in the case of saturation is shown by the unfilled points in Fig. 1. The stars and squares represent the geometric means of two experiments in which the observers were asked to assign numbers proportional to paleness. The two experiments differed mainly in the spacing of the stimuli, and both experiments included a stimulus that apneared gray, i.e., whose saturation was below threshold for redness. With the apparatus used, the threshold was near 12 or 13 per cent red in the mixture.

The general slope determined by the judgments of paleness is not greatly different from that of the dashed line, which is the negative of the slope determined by the judgments of redness. There is a tendency, however, for the function for paleness to show a downward concavity. Some degree of downward concavity has been observed on nine other continua, even for such simple judgments as the "shortness" of lines projected on a screen (Stevens \& Guirao, 1963). Observers are not, on the average, highly adept atproducing judg- 
ments in terms of reciprocal functions. Several of the observers commented on the greater difficulty of judging paleness.

It is to be noted that the question-which is the primary attribute, paleness or redness-receives a fairly clear answer in Fig. 1. The judgments of redness behave like those on other prothetic continua, whereas the judgments of paleness show the characteristics typical of inverse judgments on continua whose primary aspect (e.g., brightness as opposed to dimness) seems not to be in question.

Paleness was chosen as the name for the inverse aspect after preliminary experiments with several terms, e.g., grayness, desaturation, had shown that the word paleness directs attention to the desired attribute.

\section{Variability}

When each observer is allowed to choose his own modulus or unit for his subjective scale, the variations due to his choice of unit can be partialed out by the method of modulus equalization before measures of variability are computed. First a grand mean of the logarithms of all the judgments is obtained. A correction factor for each observer is then obtained by computing the mean of the logarithms of his estimations and subtracting this mean from the grand mean. The correction factor for each observer is added to the logarithm of each of his estimates. These operations leave unchanged the slope of the magnitude function for each observer while minimizing the sum of the squared deviations around the regression line for the group. The resulting quartile deviations of the 20 magnitude estimations for each experiment are expressed in decilogs in Table 1.

When, as in one experiment, a standard stimulus is assigned the modulus 10 , the procedure of modulus equalization usually decreases the variability less than when the observer is free to choose his own modulus. But since a given standard is perceived and remembered differently by different observers, some decrease will ordinarily be observed. In the first experiment in Table 1 the average quartile deviation was reduced from 1.34 to 1.18 decilogs when modulus equalization was applied.

Fig. 1. Magnitude estimations of saturation for redness (filled points) and paleness (unfilled points). Each point is the geometric mean of 20 judgments, two by each of ten observers. The stimuli were presented in an irregular order that was different for each observer. The duration of the stimulus was 2 sec., except for the first experiment (filled circles), in which the observer viewed each stimulus as long as he wanted to. About $10 \mathrm{sec}$. elapsed between stimuli.

The line for redness was fitted by least squares. The dashed line has the same absolute slope, 1.7, as the solid line.

\section{Cross-Modality Comparisons}

Although magnitude estimation is a convenient and fairly simple procedure for determining the function that governs the growth of a perceptual magnitude, it may be useful to verify the validity of the results by other methods. The procedure of cross-modality matching allows the observer to indicate his impression of one stimulus by adjusting a stimulus in another modality to produce a corresponding impression (Stevens, 1959). The method is essentially the same as that of heterochromatic brightness matching.

In matching loudness to saturation the observer was instructed to adjust the loudness of a band of noise (100 to $3000 \mathrm{cps}$ ) to make it seem to match the degree of redness (or paleness) of the visual stimulus. He controlled the noise by means of a pair of 2000-ohm potentiometers ganged and cascaded, which gave a smooth, wide-range variation in the sound pressure level of the noise in the earphones (PDR-8 mounted in sponge neoprene cushions $\mathrm{MX}-41 / \mathrm{AR})$.

The results of matching loudness to redness and to paleness are shown in Fig. 2. Each point is the decibel average, or geometric mean, of the sound pressure as adjusted by ten observers, two settings by each. The slope of the line fitted by least squares to the filled circles is 2.6. (One $\log$ unit of sound pressure equals $20 \mathrm{db}$.)

The next important question is how well the exponent

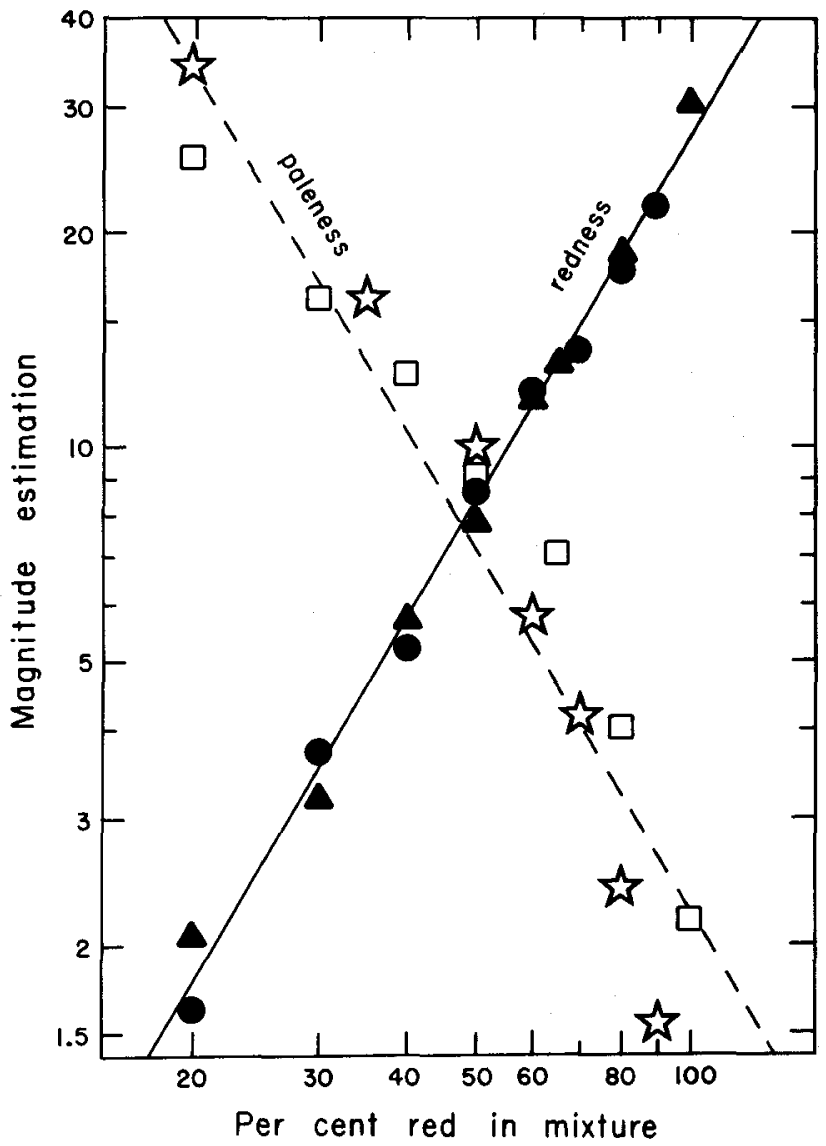




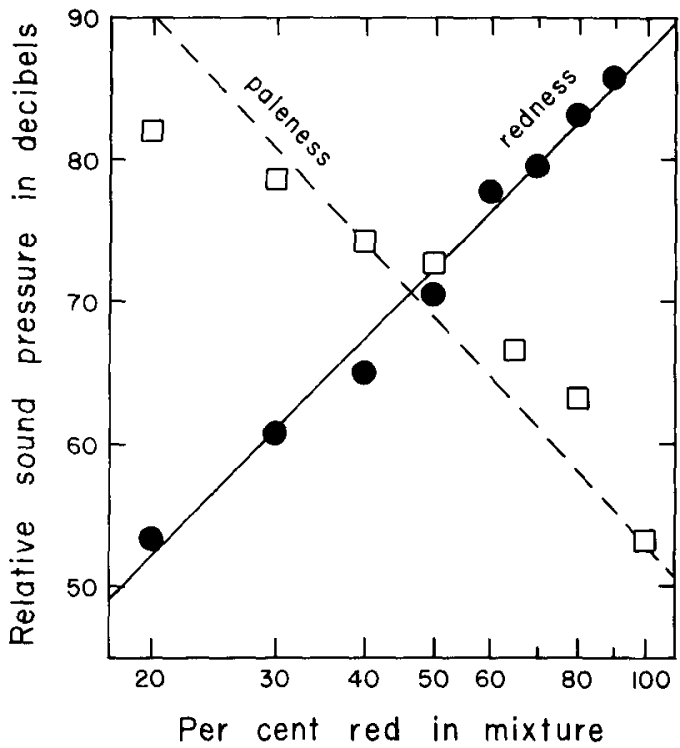

Fig. 2. Cross-modality matching of loudness to redness (filled circles) and to paleness (unfilled squares). Each point is the decibel average of two settings by each of ten observers, made in irregular order. The visual stimulus was presented for $2 \mathrm{sec}$. at a time, with a dark interval of $10 \mathrm{sec}$. The observer took as much time as he wanted for each match. The band of noise (100 to 3000 cps) was presented via earphones.

The line for redness was fitted by least squares. The dashed line has the same absolute slope, 2.6, as the solid one.

of the matching function 2.6 agrees with the exponent 1.7 obtained by direct magnitude estimation. The loudness of a tone of 1000 cps grows with an exponent of 0.6 , the value recommended for engineering calculations by the International Standards Organization. There is much accumulated evidence that the loudness of a noise of the kind used in the present experiment grows with an exponent between 0.6 and 0.7. When the exponent 2.6 is multiplied by either of these values, the result is close to the value of the exponent obtained by magnitude estimation. Looked at the other way around, the results in Fig. 1 (exponent 1.7) plus the results in Fig. 2 (matching-function exponent 2.6) suggest that the loudness of the noise grows with an exponent equal to 0.65 , which is the ratio of 1.7 to 2.6.

The matching of loudness to paleness gave results (squares in Fig. 2) roughly like those obtained from the magnitude estimations of paleness: a similar wide scatter and the same downward concavity. These results suggest again that the scaling of the inver'se aspect is beset by difficulties, regardless of whether the scaling is by numerical estimate or by cross-modality matching.

\section{Category Estimation}

Category scales are often created by asking observers to assign stimuli to a small number of categories defined by numbers or by adjectives. The categories used here were numbered 1 to 7 and the observer was asked to assign each stimulus to a category and to try to keep his category boundaries uniformly spaced. He was shown the two extreme stimuli, 1 and 7 , at the beginning of the experiment. For the experiment on redness the stimuli were $20,33,47,60,73,86$, and 100 per cent red on the color mixer. For the experiment on paleness the percentages of red on the mixer were $2,20,32,49,63,77$, and 89 . In each experiment the stimuli were presented twice each in 2-sec. exposures and in irregular order to each of ten observers.

For each stimulus the arithmetic means of the category assignments were computed. The relation between the average assignment and the percentage of red in the stimulus was found to be fairly linear, although the curve was actually slightly S-shaped. This relation of category estimation to stimulus magnitude is not, however, the question of interest. It is rather the relation of the category estimations to the subjective value of saturation, as determined by the power function in Fig. 1. Consequently, the line in Fig. 1 was used to convert the stimulus values to values of apparent redness, which were then used for the abscissa values in Fig. 3 .

Although the category scale might be expected to be a linear function of the magnitude-estimation scale, on prothetic continua it is consistently nonlinear in the manner shown in Fig. 3. Although downward concavity is a chronic ailment of category scales on prothetic continua, it is important to note that on metathetic continua the category scale is typically linear (Stevens \& Galanter, 1957)。

Figure 3 suggests that the form of the category scale is the same for paleness as for redness. This is not surprising. The task in category scaling is that of partitioning the continuum into equal-appearing intervals. In the process of partitioning, it makes little difference whether an end category is called 1 or 7 , or even whether it is named by an adjective of some kind (Stevens \& Galanter, 1957).

If the category judgments for paleness were plotted against the magnitude-estimation scale of paleness as determined by the dashed line in Fig. 1, the result would be a curve of much greater curvature than that shown by the triangles in Fig. 3. As a matter of fact, the slightly curved function in Fig. 3, plotted not against redness, bui against its approximate reciprocal, paleness, gives a function that is approximately hyperbolic.

Why is the category scale nonlinearly related to the magnitude scale on prothetic continua? Since the main functional difference between a prothetic and a metathetic continuum lies in the behavior of variability (measured in subjective units) at different points along the two continua, the answer presumably rests on this fact (Stevens \& Guirao, 1962). On prothetic continua the variability tends to grow in proportion to magnitude, i.e., the relative variability tends to be constant. On metathetic continua the absolute variability tends to be constant. The process of partitioning appears 


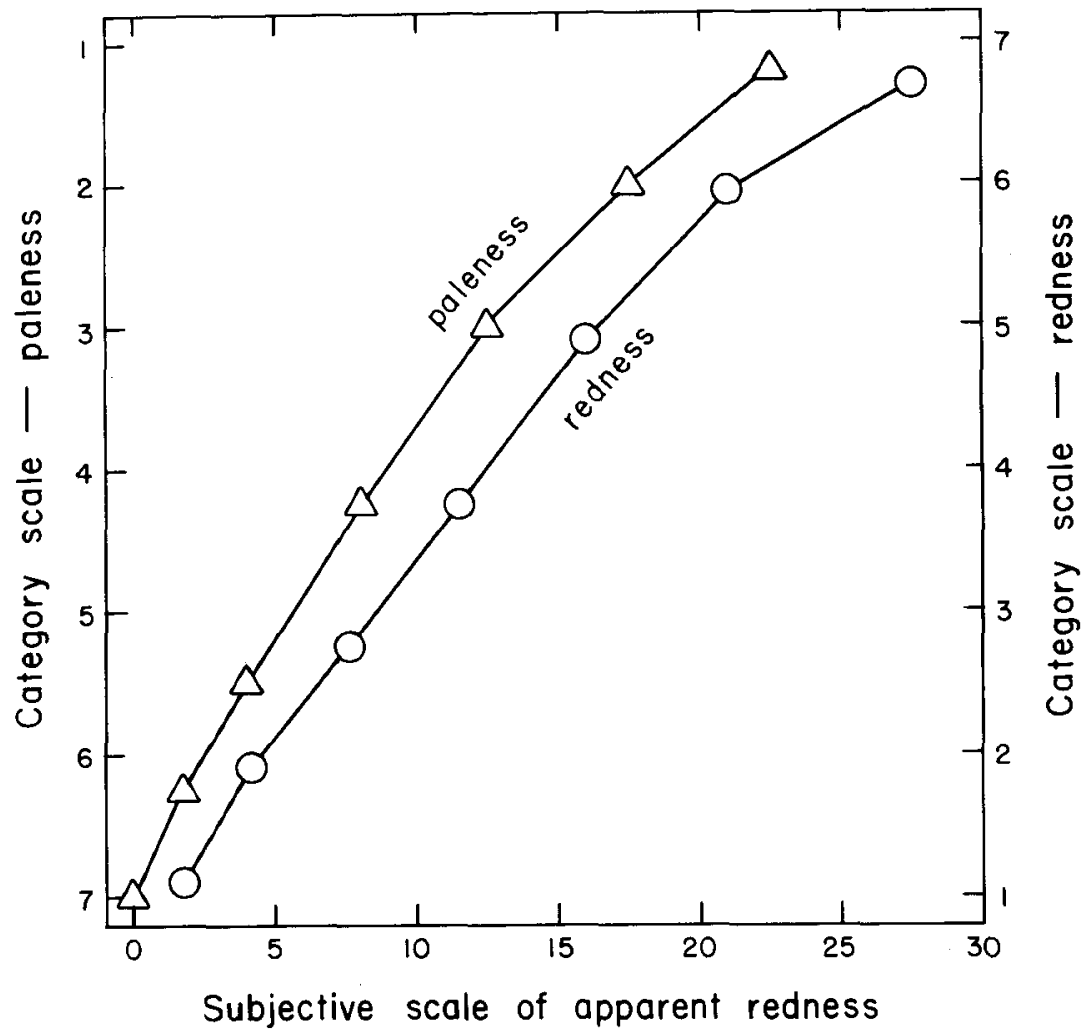

Fig. 3. The arithmetic means of the category judgments of redness (righthand ordinate) and paleness (left-hand ordinate are plotted against the subjective scale of saturation determined by the power function in Fig. 1. to be biased when variability is relative, but not when it is constant.

\section{Poikilitic Functions and the Jnd}

A useful indicator of the variability spoken of above can be had from classical jnd measurements, for a jnd, or a difference limen, is nothing more nor less than a measure of the observer's variability when he undertakes to categorize stimulus values into two or more classes, such as "more red" or "less red." This variability or "noise" is often assessed in terms of one-half the range between the stimulus that is judged more red 25 per cent of the time and the stimulus judged more red 75 per cent of the time. These values are usually read from a poikilitic function, so called because it shows the scatter or variability of the judgments (Stevens, 1965; see also J.C. Stevens \& S. S. Stevens, 1963). The poikilitic function has often been called the psychometric function, because it has been assumed that the jnd provides a unit for the psychological scale. On prothetic continua where the subjective scale is a power function, the variability, instead of remaining constant in subjective units, grows approximately in proportion to magnitude.

Measurements of the jnd for saturation were undertaken to determine how the variability behaves on this continuum and hence to determine whether saturation belongs to the class of prothetic continua.

The method of constant stimuli and a modified method of single stimuli were both used. Under the method of constant stimuli, a standard stimulus was presented for 0.5 sec., followed about 2 sec. later by one or another comparison stimulus. The observer judged the comparison to be either "more red" or "less red." At least 5 sec. elapsed between successive pairs of stimuli.

Under the method of single stimuli the standard stimulus was omitted except at the outset of each run. Although the observers were informed that they could see the standard whenever they liked, they seldom asked to see it after the experiment was in progress. As is well known, even this much use of a standard is unnecessary, for the standard can be omitted entirely with little if any change in the poikilitic function. The main effect of omitting the standard is to speed up the experiment. Under the method of single stimuli as used here, each stimulus lasted 0.5 sec., and at least 5 sec. elapsed between stimuli.

Poikilitic functions obtained under both methods are shown in Fig. 4.

The similarity of the results for the two methods led to the pooling of all judgments by the eight observers for the five center stimuli tested: $20,35,50$, 65 , and 80 per cent red. Only four observers judged the two center stimuli, 35 and 50 per cent red. The proportions of "more red" judgments were plotted on probability paper, and straight lines were drawn through the points by eye. Half the distance between the 25- and the 75-per-cent values was then measured. Converted to stimulus units, these distances give as jnd the values $1.6,2.4,2.5,3.0$, and 3.2 percentage 

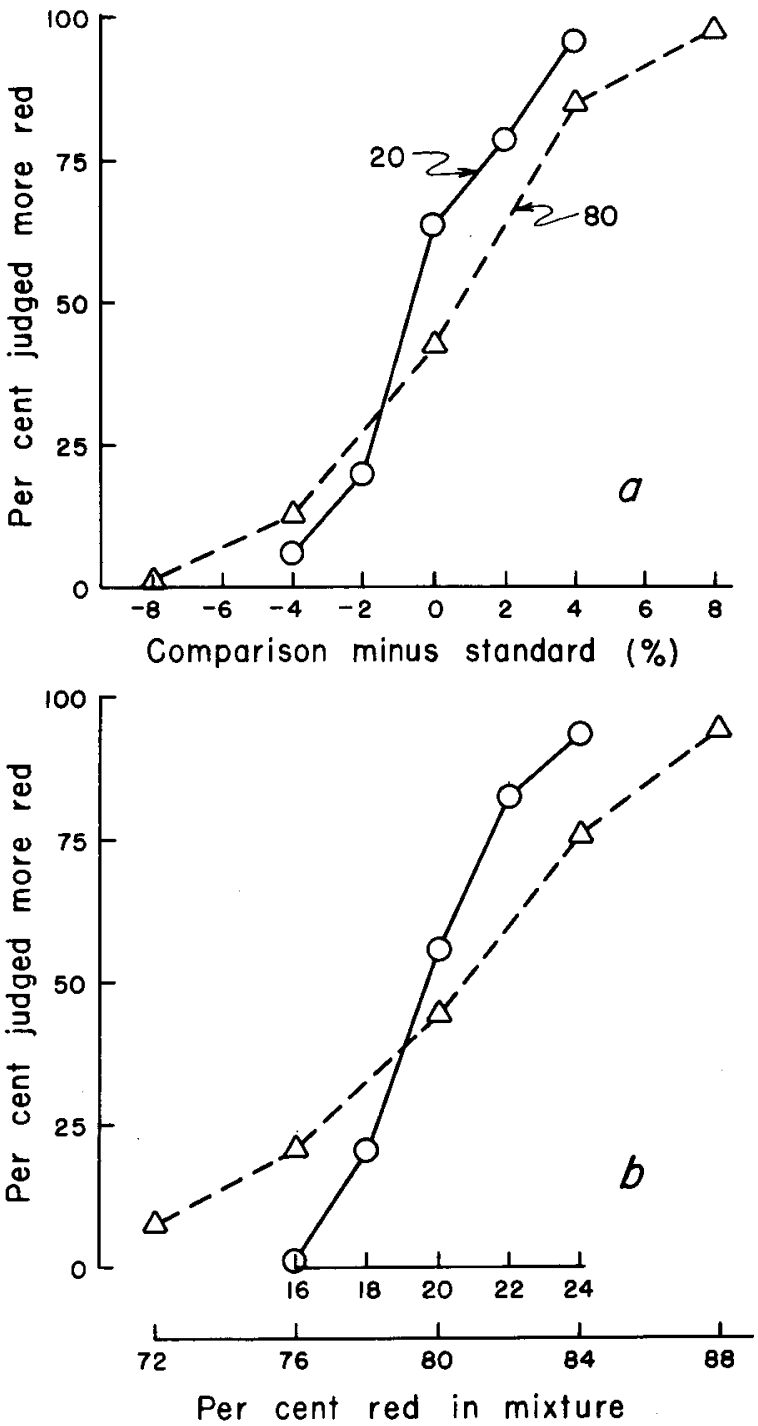

Fig. 4. Samples of the poikilitic functions obtained by the methods of constant stimuli (a) and single stimuli (b). By the method of constant stimuli, four observers each made 20 judgments of each comparison stimulus for two different standards, 20 and 80 per cent as shown in a. By the method of single stimuli, four other observers each made 30 judgments of each of the stimuli designated by the two abscissa scales in $b$.

points of red. A plot of these values shows that they give only a fair approximation to Weber's law in its general form: $Q=k S+C$, where $Q$ is the quartile deviation (the jnd as computed here), $S$ is the stimulus magnitude, and $k$ and $C$ are constants.

The use of the symbol $Q$ instead of $\Delta S$ is deliberate. The symbol $\Delta S$ (or $\Delta I$ ) has led to much misunderstanding, for what these measurements are concerned with is a dispersion, not an increment. If all noise, variability, or dispersion could be eliminated, the poikilitic functions in Fig. 4 would become step functions and the resolving power of the system would appear to be infinitely good. Under some experimental arrangements it has been possible to reduce certain components of the variability to the point where evidence for an underlying step function can be seen (Luce, 1963; Stevens, 1961a).

In order to demonstrate the prothetic character of saturation, it is necessary to convert the jnd measures into subjective units. This was done with the aid of the power function in Fig. 1. The results are plotted in Fig. 5, where both coordinates are subjective values. Two points are evident in Fig. 5. The variability $\mathrm{Q}_{\psi}$ (the subjective jnd) increases in magnitude as $\psi$ (the apparent redness) increases. In this respect. redness behaves as a prothetic continuum. The other feature is the linearity of the function. These results confirm Brentano's suggestion that a law similar to Weber's may hold for subjective magnitudes (Stevens, 1957, 1961b). Many instances of linear equations like that shown in Fig. 5 have been presented by Ekman (1961), who has done much to show the generality of the relation. Just as Weber's law describes how variability behaves in stimulus terms, Ekman's law tells how variability behaves when measured in subjective units.

One of the important consequences of Ekman's law is to bring into question the scaling of saturation by the well-known procedure of counting off jnd (Troland, 1930). When the relation conjectured by Brentano holds, the jnd does not provide an invariant unit. Rather it provides a kind of rubber unit that stretches to a progressively larger size as the sensory magnitude increases. The stretching is plain enough in Fig. 5, but the over-all change in the subjective size of the jnd for saturation appears to be much smaller than that observed on perceptual continua that have greater dynamic ranges, such as loudness and brightness. Discussion

A promising consensus appears to have been reached among recent experiments as regards the power-law relation between purity and apparent saturation. Saturation can be added to the long list of continua on which equal stimulus ratios produce equal sensation ratios. This ratio invariance is the essence of the psychophysical power law (Stevens, 1957).

In at least four independent experiments, the value of the exponent for saturation (redness) has been found to lie in the general vicinity of $2.0 \pm 0.3$. The value of the exponent seems to depend upon the parameters of the experiment. Thus Onley et al (1963), using mixtures of filtered light rather than colored papers, measured redness exponents that depended on the visual angle subtended by the stimulus: a target of 4 degrees gave exponents of 2.3 and 2.2 ; a target of 1.5 degrees gave exponents of 1.8 and 1.9. One of the present authors has shown that the saturation of a red-green mixture depends rather strongly on the size of the retinal image (Stevens, 1934), but the relation of that fact to the exponent of the power function has not been explored. 


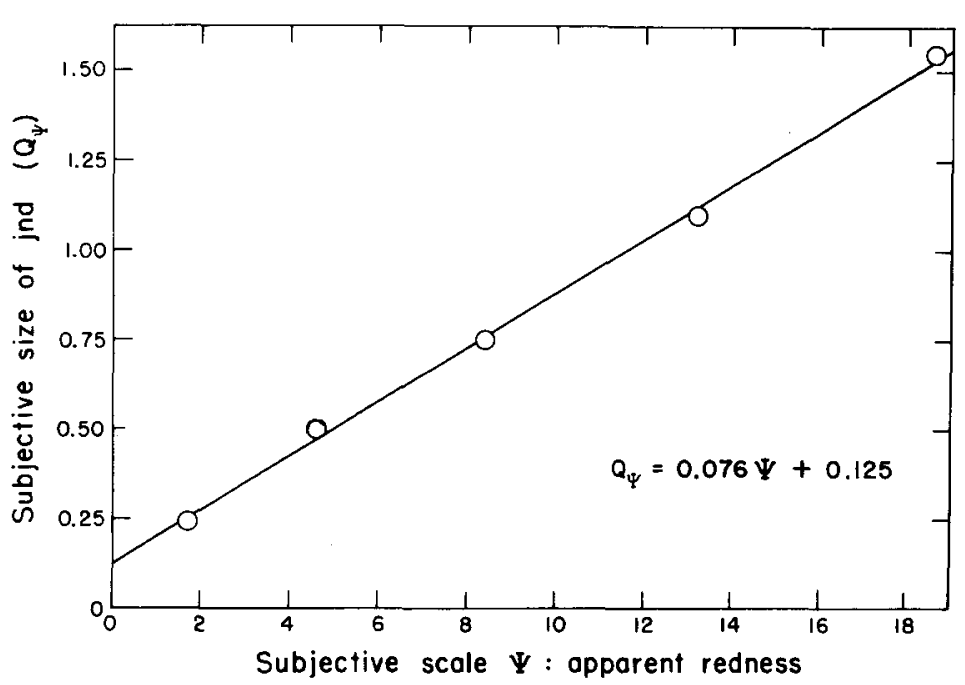

Fig. 5. The subjective size of the jnd is shown as a function of the subjective magnitude produced by the stimulus about which the dispersion was measured. The rising linear function suggests that saturation (iedness) is a prothetic continuum.
Additional experiments have been conducted in this laboratory by Tarow Indow with the aid of a rotating optical prism that made it possible to mix filtered lights in any proportions, and, when desired, to place the control of the mixture in the hands of the observer. Using red light and the method of magnitude estimation, Indow has obtained a power function from fourteen observers, giving an exponent of 1.7. Thus there is fair agreement among the results of Onley and her collaborators, the present authors, and Indow. Indow has also shown that the exponent for saturation varies with wavelength.

The earlier effort by Galifret (1959) to scale saturation involved two procedures, ratio production (both halving and doubling) and magnitude estimation. Thirty observers adjusted a saturation to appear "half as red" as the standards made up of $35,50,65$, and 80 per cent red on a cylindrical color mixer. They also produced "twice as red" for the standards $20,35,50$, and 65 per cent red. Each observer made five settings for each standard. If apparent saturation is a power function of per cent red, the ratio of the lesser to the greater stimulus for each setting should be the same. The pooled ratios for both halving and doubling for the five standards, 20 to 80 per cent, are $0.59,0.66$, $0.69,0.73$ and 0.74 . These ratios are not quite constant, but neither do they vary greatly. If their average value 0.68 is used to compute the exponent for a power function, its value turns out to be 1.8, an exponent that accords fairly well with the values found in the more recent experiments.

Galifret's experiment with magnitude estimation, undertaken at the suggestion of one of the present authors, gave results that approximate a power function with an exponent of only about 1.0. This low value may have been due to the failure of the author of the suggestion to make it clear that only a brief sample of judgments (usually 2 per stimulus) need be taken from each observer. Since the observers soon learned to identify the stimuli on repeated trials, Galifret tried to make the identification more difficult by interpolating additional stimuli. The results for these interpolated stimuli were later discarded.

Whatever the cause, the results of Galifret's second experiment stand as the one exception to an otherwise fairly consistent set of findings by the experimenters who have used one or another method of direct judgment to scale saturation.

One further matter deserves comment. In several of the sets of data now available, there is a slight tendency for the general course of the data to be concave upward when plotted in log-log coordinates. This behavior sets saturation apart from most other prothetic continua, where the tendency is for the functions to turn downward at their lower ends (J. C. Stevens \& S. S. Stevens, 1963). The downward curvature near threshold has led to a formulation of the psychophysical law in terms of a stimulus measure $\phi$ that starts at the threshold value $\phi_{0}$. Thus the psychophysical magnitude $\psi$ may be related to the physical magnitude $\phi$ by $\psi=\mathrm{k}(\phi-\phi) \beta \quad$ where $\mathbf{k}$ depends on units and $\beta$ is the exponent. Clearly this general form of the psychophysical power law does not accord precisely with what is usually seen near the bottom end of the scale of saturation, and we are led to ask why.

No assured reason can be given, but one possibility may be suggested. When the purity of a stimulus is decreased, the redness does not fade away in the same absolute sense that a brightness fades away when luminance is decreased to threshold. It is as though the redness were being replaced by something else -something equally plain and visible. It is perhaps more like a changing proportion than like an extinction. In the face of the emerging paleness of the stimulus, it may be that some observers lose their grip on the quantitative aspect of the redness that they are trying to abstract from the configuration. In any case the resulting bias remains small in the sense that it does not greatly disturb the fit of the data to a power function, as is shown in Fig. 1. 


\section{Summary}

A battery of scaling procedures was applied to the visual saturation of colors produced by mixtures of red and gray papers. By direct magnitude estimation, the apparent saturation was found to grow as the 1.7 power of the percentage of red in the mixture. The power law was confirmed by the cross-modality matching of loudness to saturation, and the matching-function exponent had the predicted value, namely, a value approximately equal to the ratio between the exponents for saturation and loudness. The foregoing procedures were also used to scale the inverse continuum, paleness.

Three findings suggest that saturation behaves as a prothetic (intensive) continuum and not as a metathetic (qualitative) continuum: (1) like brightness, loudness, and other prothetic continua, the apparent magnitude grows as a power function of the stimulus magnitude; (2) the category (partition) scale is nonlinear relative to the scale of direct magnitude estimation; and (3) the just noticeable difference, measured in subjective units, grows larger as saturation increases.

\section{Reforences}

Ekman, G. Some aspects of psychophysical research. In W. A, Rosenblith(Ed.), Sensory communication. New York: M.I.T. Press and Wiley, 1961. Pp. 35-48.

Galifret, Y. Les psychophysiques de la saturation chromatique. Année psychol., 1959, 59, 35-46.

Luce, R. D. Detection and tecognition. In R. D. Luce, R. R. Bush, and E. Galanter (Eds.), Handbook of mathematical psychology. Vol. I. New York: Wiley, 1963. Pp. 157-165.

Onley, J. W., Klingberg, C. L., Dainoff, M. J., \& Rollman, G. B. Quantitative estimates of saturation. J. Opt. Soc. Amer., 1963. 53, 487-493.
Stevens, J. C., \& Stevens, S. S. Brightness function: Effects of adaptation. J. Opt. Soc. Amer., 1963, 53, 375-385.

Stevens, S. S. The relation of saturation to the size of the retinal image. Amer. J. Psychol., 1934, 46, 70-79.

Stevens, S. S. On the problem of scales for the measurement of psychological magnitudes. J. unif. Sci. (Erkenntnis), 1939, 9, 94-99.

Stevens, S. S. On the psychophysical law. Psychol. Rev., 1957, 64, 153-181.

Stevens, S. S. Problems and methods of psychophysics. Psychol. Bull., 1958, 55, 177-196.

Stevens, S. S. Cross-modality validation of subjective scales for loudness, vibration, and electric shock. J. exp. Psychol.. 1959, 57, 201-209.

Stevens, S. S. Is there a quantal threshold? In W. A. Rosenblith (Ed.), Sensory communication. New York: M.I.T. Press and Wiley, 1961a. Pp. 806-813.

Stevens, S. S. To honor Fechner and repeal his law. Science, 196 ih 133, 80-86.

Stevens, S. S. On the uses of poikilitic functions. In D. J. Mostofsky (Ed.), Stimulus generalization. Stanford, Calif.: Stanford University Press, 1965. Pp. 24-29.

Stevens, S. S., \& Galanter, E. H. Ratio scales and category scales for a dozen perceptual continua. J. exp. Psychol., 1957, 54, 377. 411.

Stevens, S. S., \& Guirao, Miguelina. Loudness, reciprocality, and partition scales. J. Acoust. Soc. Amer., 1962, 34, 1466-1471.

Stevens, S. S., \& Guirao, Miguelima. Subjective scaling of length and area and the matching of length to loudness and brightness. J. exp. Psychol., 1963, 66, 177-186.

Stevens, S. S., \& Volkmann, J. The relation of pitch to frequency: A revised scale. Amer. J. Psychol., 1940, 53, 329-353.

Troland, L. T. The principles of psychophysiology. Vol. 2, New York: Van Nostrand, 1930.

\section{Note}

1. This investigation was supported in part by grants from the National Science Foundation and the National Institutes of Health, and in part by Public Health Service Fellowship MPM-12,800 (Laboratory of Psychophysics Report PPR-303). 